\title{
Solar Dynamics Observatory On-orbit Jitter Testing, Analysis, and Mitigation Plans
}

\author{
Kuo-Chia (Alice) Liu ${ }^{1}$ \\ NASA Goddard Space Flight Center, Greenbelt, MD, 20771 \\ Carl A. Blaurock ${ }^{2}$ \\ Nightsky Systems Inc., Raleigh, NC, 27616 \\ Kristin L. Bourkland ${ }^{3}$, Wendy M. Morgenstern ${ }^{4}$, and Peiman G. Maghami ${ }^{5}$ \\ NASA Goddard Space Flight Center, Greenbelt, MD, 20771
}

\section{Introduction}

$\mathrm{T}_{\mathrm{b}}$

he Solar Dynamics Observatory (SDO) was designed to understand the Sun and the Sun's influence on Earth. SDO was launched on February 11, 2010 carrying three scientific instruments: the Atmospheric Imaging Assembly (AIA), the Helioseismic and Magnetic Imager (HMI), and the Extreme Ultraviolet Variability Experiment (EVE). Both AIA and HMI are sensitive to high frequency pointing perturbations and have sub-arcsecond level line-of-sight (LOS) jitter requirements. Extensive modeling and analysis efforts were directed in estimating the amount of jitter disturbing the science instruments. To verify the disturbance models and to validate the jitter performance prior to launch, many jitter-critical components and subassemblies were tested either by the mechanism vendors or at the NASA Goddard Space Flight Center (GSFC).

Although detailed analysis and assembly level tests were performed to obtain good jitter predictions, there were still several sources of uncertainties in the system. The structural finite element model did not have all the modes correlated to test data at high frequencies $(>50 \mathrm{~Hz})$. The performance of the instrument stabilization system was not known exactly but was expected to be close to the analytical model. A true disturbance-to-LOS observatory level test was not available due to the tight schedule of the flight spacecraft, the cost in time and manpower, difficulties in creating gravity negation systems, and risks of damaging flight hardware. To protect the observatory jitter performance against model uncertainties, the SDO jitter team devised several on-orbit jitter reduction plans in addition to reserve margins on analysis results. Since some of these plans severely restricted the capabilities of several spacecraft components (e.g. wheels and High Gain Antennas), the SDO team performed on-orbit jitter tests to determine which jitter reduction plans, if any, were necessary to satisfy science LOS jitter requirements. The SDO on-orbit jitter tests were designed to satisfy the following four objectives:

1. Determine the acceptable reaction wheel operational speed range during Science Mode.

2. Determine HGA algorithm jitter parameters (number of stagger steps and enable/disable no-steprequests).

3. Determine acceptable EVE instrument filter wheels spin rates.

4. Determine if AIA instrument filter wheels excite the first AIA telescope structural mode.

This paper provides detailed information on the SDO wheel jitter test plan, shows on-orbit jitter measurements and how ground predictions compare to those measurements, and describes the final jitter mitigation plan executed on SDO.

\section{Reaction Wheel Jitter Tests}

Based on pre-flight jitter analysis estimates, the wheel speeds were limited to $\pm 400 \mathrm{rev} / \mathrm{min}$ (RPM) to meet AIA and HMI jitter requirements. This constraint forces the wheels to reverse direction about once every four weeks and uses only a small portion of the available wheel speed range of $\pm 6000 \mathrm{RPM}$. As the wheel reverses direction, a small increase in LOS motion would occur and was considered an acceptable error to the science team. However,

\footnotetext{
${ }^{1}$ Aerospace Engineer, Attitude Control System Engineering Branch, Greenbelt MD, 20771, AIAA Lifetime Member.

${ }^{2}$ President, Nightsky Systems Inc., 3916 Lauriston Rd, Raleigh NC 27616, AIAA Member.

${ }^{3}$ Aerospace Engineer, Attitude Control System Engineering Branch, Greenbelt MD, 20771.

${ }^{4}$ Aerospace Engineer, Attitude Control System Engineering Branch, Greenbelt MD, 20771, AIAA Member.

${ }^{5}$ Aerospace Engineer, Attitude Control System Engineering Branch, Greenbelt MD, 20771, AIAA Member. 1

American Institute of Aeronautics and Astronautics
} 
the team preferred to reduce the frequency of wheel reversals and the number of thruster maneuvers required to unload wheel momentum. The objective of the wheel jitter tests is to measure the actual wheel-induced jitter on orbit, which is expected to be smaller than the analytical prediction. The on-orbit jitter measurements allowed the SDO team to extend the wheel speed jitter limit to +/- 800 RPM and thereby reduce the number of direction reversals each wheel will experience over its lifetime and prolong the period between thruster momentum unloads.

\section{High Gain Antenna (HGA) Jitter Tests}

The goal of the HGA tests is to characterize the jitter induced by HGA operation, and if possible remove constraints on HGA operation if induced jitter is sufficiently low. The objective is to operate the HGA actuators in all four gimbal axes for a range of representative pulse rates to determine the conservatism in the pre-flight jitter predictions.

Pre-flight jitter analysis demonstrated that the peak jitter occurred when steps from multiple actuators interfered constructively. In rare cases, this interference resulted in a doubling of induced jitter. To avoid this phenomenon, a stagger-step algorithm was implemented to prevent actuators on the $+Z$ and $-Z$ antennas from taking steps during the same 200-ms HGA control cycle. Even with the stagger-step implementation, high jitter was still predicted to occur very infrequently (less than $1 \%$ of the time) and for short durations. AIA was relatively insensitive to this effect, since the resulting image loss could fit within the imaging data continuity budget. However, HMI has a registration requirement that all of the images in a sequence must be taken in a suitable jitter environment. Therefore, the peak jitter was not acceptable. The operational constraint imposed in order to meet HMI requirements took the form of a No Step Request (NSR) flag, sent from HMI to the ACS system, which requests the ACS not to move any of the HGAS gimbals after a specified period for a specified duration.

The HGA jitter tests showed flight measurements were higher than pre-flight predictions at various HGA joint configurations, despite several levels of conservatism, on assumed damping, with large margins used in the jitter analysis. As a result, HGA jitter mitigation options (stagger-stepping and NSR flags) were enabled to meet instrument LOS jitter requirements. The mitigation algorithms worked as expected and greatly reduced HGAinduced jitter in science images.

\section{EVE Instrument Filter Wheel Jitter Test}

The EVE instrument does not have tight jitter requirements, but it contains four filter wheels (FW) driven by stepper motors. The EVE FW are also large disturbance contributors after the HGAs and the reaction wheels, but they do not operate as frequently as the other two jitter sources. Each EVE FW is expected to move only a few seconds per day in a 40-min window. Although the FW move infrequently, the HMI science data can still be significantly impacted if the EVE FW generate large jitter disturbances. For example, a short period (e.g. seconds) of larger jitter can cause HMI to lose an entire 4-minute data record. The HMI data continuity requirement may not be met if HMI loses one or two 4-minute data records every day due to EVE FW movements. Based on pre-flight jitter analysis, large jitter disturbances are expected to occur only if the EVE FW step rate excites one of the observatory structural modes. Since the EVE FW step rate may be tuned on-orbit, the jitter team varied the step rate and determined which rate should be used to minimize FW-induced jitter to all SDO instruments.

The EVE jitter tests demonstrated that a lower EVE step rate $(91 \mathrm{pulses} / \mathrm{sec})$ introduced less jitter than the nominal step rate $(125 \mathrm{pulses} / \mathrm{sec})$. However, the EVE FW-induced jitter operating at the nominal rate was acceptable to all instruments. Additionally, since the FW environmental and functional tests were performed at the nominal step rate, the SDO team decided to maintain the FW step rate and avoided using a new step rate with fewer ground tests.

\section{AIA Filter Wheel Jitter Test}

Each of the four AIA telescopes employs a filter wheel. The AIA FW is operated by a stepper motor with a fixed commutation frequency set at $71.3 \mathrm{~Hz}$. From the SDO finite element model, two structural modes $(69.2$ and $70.36 \mathrm{~Hz}$ ), associated with bending modes of the AIA telescopes, were noted to be close to the AIA FW commutation frequency. AIA FW induced disturbances were well within their jitter allocations for the HMI LOS. However, larger AIA LOS jitter could be generated if one or more actual observatory modes are close to the AIA FW commutation frequency. The SDO team designed the AIA FW jitter test to verify that the FW-induced jitter is acceptable to all instruments as predicted by pre-flight jiter anlaysis, This knowledge would assist the AIA team in designing their observation sequences to minimize the effects of AIA FW disturbances.

Fortunately, the on-orbit jitter measurements showed AIA FW contributed acceptable jitter level to all other instruments. No additional mitigation plan was required to reduce AIA FW disturbances.

2

American Institute of Aeronautics and Astronautics 